\title{
Next Generation Sequencing in Pediatric Epilepsy Using Customized Panels: Size Matters
}

\author{
Eva-Katharina Willimsky ${ }^{1}$ Anna Munzig ${ }^{2}$ Karin Mayer ${ }^{2}$ Saskia Biskup ${ }^{3}$ Angela Abicht ${ }^{4}$ \\ Konstanze Hoertnagel ${ }^{2}$ Hubertus von Voss ${ }^{2}$ Hanns-Georg Klein ${ }^{2}$ Imma Rost ${ }^{2}$ Line H.G. Larsen ${ }^{5}$ \\ Hanns Atli Dahl ${ }^{5}$ Hannes Hoelz ${ }^{1}$ Celina von Stuelpnagel ${ }^{1,6}$ Ingo Borggraefe ${ }^{1,7}$
}

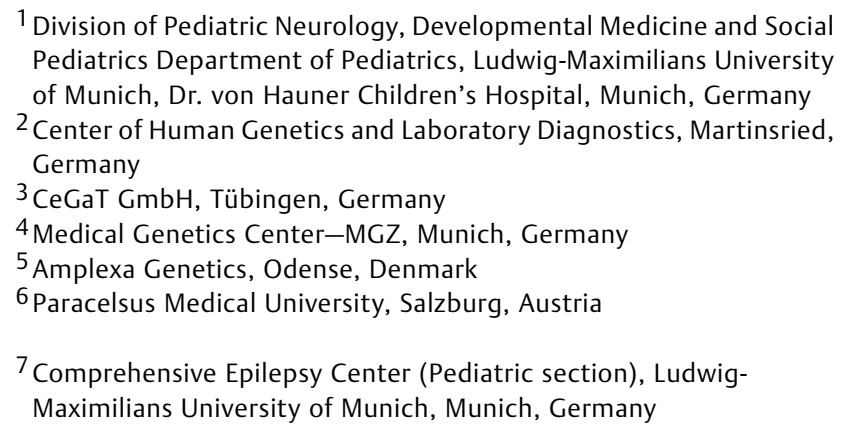

Address for correspondence Ingo Borggraefe, MD, Division of Pediatric Neurology, Developmental Medicine and Social Pediatrics, Department of Pediatrics, University of Munich, Dr. von Hauner Children's Hospital, Lindwurmstrasse 4, 80337 Munich, Germany (e-mail: ingo.borggraefe@med.uni-muenchen.de).

Neuropediatrics 2021;52:92-97.

\begin{abstract}
Introduction Next generation sequencing (NGS) with customized gene panels is a helpful tool to identify monogenic epilepsy syndromes. The number of genes tested within a customized panel may vary greatly. The aim of the present study was to compare the diagnostic yield of small ( $<25 \mathrm{~kb}$ ) and large $(>25 \mathrm{~kb}$ ) customized epilepsy panels.

Methods This retrospective cohort study investigated data of 190 patients of 18 years or younger, with the diagnosis of an epilepsy of unknown etiology who underwent NGS using customized gene panels. Small $(<25 \mathrm{~kb})$ and large $(>25 \mathrm{~kb})$ panels were compared regarding the distribution of benign/likely benign and pathogenic/likely pathogenic variants and variants of unclear significance. In addition, differences of the diagnostic yield with respect to epilepsy severity, i.e., developmental and epileptic encephalopathy [DEE] vs. non-DEE, were analyzed.

Results The diagnostic yield defined as pathogenic or likely pathogenic variants in large panels was significantly increased $(29 \%[n=14 / 48]$ vs. $13 \%[n=18 / 142]$, $p=0.0198)$ compared with smaller panels. In non-DEE patients the increase of the diagnostic yield in large panels was significant(35\% $n=6 / 17$ vs. $13 \% n=12 / 94$,

Keywords

- next generation sequencing

- NGS panel

- pediatric epilepsy

- customized panel

- panel size

- yield $p=0.0378$ ), which was not true for DEE patients.

Discussion This study indicates that large panels are superior for pediatric patients with epilepsy forms without encephalopathy (non-DEE). For patients suffering from DEE small panels of a maximum of 10 genes seem to be sufficient. The proportion of unclear findings increases with rising panel sizes.

Conclusion Customized epilepsy panels of $>25 \mathrm{~kb}$ compared with smaller panels show a significant higher diagnostic yield in patients with epilepsy especially in non-DEE patients.
\end{abstract}

received

February 9, 2020

accepted

April 7, 2020

published online

October 21, 2020 (c) 2020. Thieme. All rights reserved.

Georg Thieme Verlag KG,

Rüdigerstraße 14,

70469 Stuttgart, Germany
DOI https://doi.org/

10.1055/s-0040-1712488. ISSN 0174-304X. 


\section{Introduction}

Next generation sequencing (NGS) is a helpful tool to identify monogenic epilepsy syndromes by scanning the deoxyribonucleic acid (DNA) for genetic causes. NGS has increased the finding of pathogenic DNA variants, defined as diagnostic yield.

Targeted NGS panels include several genes that are associated with a certain epileptic phenotype. Panels may vary significantly in size, which might be due to coverage of costs of these investigations in a certain heath system. For instance, gene panels up to $25 \mathrm{~kb}$ coding sequence (this translates roughly into approximately 10 genes depending on the size of the genes of interest) are covered by German Public Health Insurance. However, tests of panels comprising 100 genes and more are also applied and are either covered by private insurances or self-paid by the patients. ${ }^{1}$ Although it might seem obvious that the yield of the investigation might increase with the numbers of genes tested, there is only sparse data to support this relationship. In addition, the number of detected variants of unknown significance (VUS), as defined by the ACMG (American College of Medical Genetics), may increase with the panel size potentially creating confusion within the genetic consultation for the patients and parents. ${ }^{2,3}$

Hundreds of genes are known to cause monogenetic epilepsies and within the last decade; ongoing research frequently identified new epilepsy-associated genes. ${ }^{4}$ More than 30 different genes are known to cause developmental and epileptic encephalopathies (DEEs). ${ }^{5}$ DEE comprises severe epileptic syndromes such as West syndrome, Dravet syndrome, Ohtahara syndrome, and others. It is characterized by refractory seizures often commencing at the age of 6 months or younger, global developmental delay, progressive decline of cognitive function, and severe electroencephalography (EEG) abnormalities. ${ }^{6}$ Especially in these cases early diagnosis may omit potentially harmful tests, reduce overall costs, and influence therapeutic approaches. ${ }^{7,8}$

The present multicenter, retrospective cohort study investigates and compares the diagnostic yield of customized small $(<25 \mathrm{~kb})$ and large $(>25 \mathrm{~kb})$ NGS panels with relation to the clinical phenotype (i.e., DEE vs. non-DEE)

\section{Methods}

NGS results of 190 consecutive epilepsy patients aged 18 and younger, tested between October 2013 and April 2018 by four different genetic laboratories (Center of Human Genetics and Laboratory Diagnostics, Martinsried, Germany; CEGAT Tübingen, Germany; Amplexa Genetics, Odense, Denmark, and Medical Genetics Center Munich, Germany), were reviewed retrospectively. The stratification of DEE and non-DEE patients was established by reviewing the clinical data by experienced clinical epileptologists (I.B. and C.V.S.). Demographic, clinical, and genetic data were spread into an excel data sheet for documentation and analysis after obtaining the informed consent of the patients or their legal representatives for participation. This study was approved by the local ethical board of the Medical Faculty of the University of Munich (No. 18-232) and was performed according to recommendations of the actual revision of the declaration of Helsinki.

All genomic changes reported by the laboratories were rated using the categories of the ACMG classification system: "benign," “likely benign," "uncertain significance," “likely pathogenic" and "pathogenic." 3 The distribution of these categories was analyzed for each subset of panels. The diagnostic yield was stated in percent and defined by the proportion of panels with likely pathogenic and pathogenic variants found in all panels performed in total (panels with variants of all categories plus panels without any variant found).

Patients were divided into two cohorts. A group of patients with small panels comprising $25 \mathrm{~kb}$ and less and a group of patients with large panels comprising more than $25 \mathrm{~kb}$. For each patient the performed targeted NGS panel and the number of genes tested was recorded. Further criteria analyzed were clinical data such as family history, developmental delay, MRI outcome, the suspected epilepsy diagnosis, the age of onset, and the time between clinical onset and genetic testing. All details were recorded into an Excel data sheet.

The differences with respect to occurrence of developmental delay, yield of pathogenic or likely pathogenic variants (total cohort and cohort divided in non-DEE and DEE patients), and the distribution of variants of unclear significance of small and large panels were compared using the Fisher's exact test. The differences in the age of onset between the small and large panel cohort were calculated using the $t$-test for unpaired samples. We used the Benjamini-Hochberg procedure to decrease the false discovery rate and to minimize the bias of multiple testing. ${ }^{9}$ Consequently, we stated only the adjusted $p$-values within the whole manuscript. We suggested statistical significance when adjusted $p$-values were below 0.05 (two-tailed).

For some patients several variants of different pathogenicity level were identified. In these cases, the most severe variant was used in categorizing the outcome of a patient's panel.

Furthermore, for single genes, the frequency of testing and the yield were investigated across all panels in the small and the large panel cohort.

\section{Results}

In this retrospective cohort study we included a total of 190 patients (102 female, 88 male) undergoing NGS diagnostic.

In 142 cases $(74.7 \%)$, a small panel $(<25 \mathrm{~kb})$ test was performed with a mean number of $6.2( \pm 3.1,1-10)$ tested genes. Forty-eight cases (25.3\%) were made up by large panels $(>25 \mathrm{~kb})$ with a mean number of $88.2( \pm 16.4$, 62-118) genes tested. Patients in the large panel cohort were younger (adjusted $p=0.0046$ ) at the onset of the epileptic symptoms, $1.9 \pm 2.8$ standard deviation (SD) years (range $0-11$ years) versus $4.4 \pm 4.3$ SD years (range $0-17$ years). In addition, patients in the large panel cohort showed a higher rate of developmental delay ( 80 vs. $53 \%$, adjusted $p=0.0046$; - Table 1 ).

Regarding all genes tested separately across all panels, the gene covered most frequently by both small and large panels was SCN1A. In this gene most pathogenic variants were found. 
Table 1 Demographic data, type of epilepsy, and genetic outcome of the small and large panel cohort

\begin{tabular}{|c|c|c|c|}
\hline \multirow[t]{2}{*}{ Total patients } & & $\begin{array}{l}\text { Small } \\
(<25 \mathrm{~kb})\end{array}$ & $\begin{array}{l}\text { Large } \\
(>25 \mathrm{~kb})\end{array}$ \\
\hline & & 142 & 48 \\
\hline Age of onset in years & Mean ( \pm SD, Min-Max) & $4.4( \pm 4.3,0.0-17.9)$ & $1.9( \pm 2.8,0.0-11.8)$ \\
\hline \multirow[t]{2}{*}{ Developmental delay } & Yes & $n=42$ & $n=37$ \\
\hline & No & $n=37$ & $n=9$ \\
\hline Number of genes tested & Mean ( \pm SD, Min-Max) & $6.2( \pm 3.1,1-10)$ & $88.2( \pm 16.4,62-118)$ \\
\hline \multirow[t]{2}{*}{ Gender } & Male & $n=69$ & $n=19$ \\
\hline & Female & $n=73$ & $n=29$ \\
\hline \multirow[t]{3}{*}{ Epilepsy classification } & DEE & $n=32$ & $n=30$ \\
\hline & Non-DEE & $n=94$ & $n=17$ \\
\hline & Not further specified & $n=16$ & $n=1$ \\
\hline Proportion of VUS & & $7 / 142(5 \%)$ & $25 / 48(52 \%)$ \\
\hline $\begin{array}{l}\text { Yield of single genes: number } \\
\text { affected/number tested }\end{array}$ & & $\begin{array}{l}\text { SCN1A: } 6 / 88(7 \%) \\
\text { KCNQ2: } 4 / 33(12 \%) \\
\text { SCN2A: } 3 / 51(6 \%) \\
\text { STXBP1: } 1 / 11(9 \%) \\
\text { CACNA1A: } 1 / 16(6 \%)\end{array}$ & $\begin{array}{l}\text { SCN1A: 4/51 (8\%) } \\
\text { CACNA1A: } 2 / 50(4 \%) \\
\text { SCN8A: } 1 / 51(2 \%) \\
\text { SCN2A: } 1 / 51(2 \%) \\
\text { KCNQ2: } 1 / 51(2 \%)\end{array}$ \\
\hline
\end{tabular}

Abbreviations: DEE, developmental and epileptic encephalopathy; SD, standard deviation; VUS, variants of unknown significance.

An overview about demographic data, epilepsy classification, and genetic findings gives - Table 1. Epilepsy classification of the DEE patients $(n=62)$ was as follows: Dravet syndrome ( $n=13)$, West syndrome $(n=11)$, myoclonic epilepsy $(n=13)$, atypical benign partial epilepsy $(n=2)$, Landau Kleffner syndrome $(n=2)$, epileptic encephalopathy with continuous spike and wave during sleep $(n=2)$, Rett-like syndrome $(n=1)$, astatic atonic epilepsy $(n=1)$, Ohtahara syndrome $(n=1)$, and epileptic encephalopathy without further specification $(n=16)$.

Epilepsy classification in non-DEE patients $(n=111)$ was as follows: idiopathic generalized epilepsy $(n=47)$, focal epilepsy $(n=32)$, generalized epilepsy with febrile seizures plus, GEFS $+(n=17)$, idiopathic focal epilepsy $(n=9)$, neonatal seizures $(n=3)$, and multiregional epilepsy $(n=3)$. In 17 further patients, a further distinction in DEE or non-DEE was not possible.

The proportion of pathogenic and likely pathogenic genes was $13 \%$ in small panels to $29 \%$ in large panels (adjusted $p=0.0198$; - Fig. 1). The diagnostic yield of large panels compared with small panels was significantly higher in nonDEE patients (35\%, $n=6 / 17$ vs. $13 \% n=12 / 94$, adjusted $p=0.0378)$, which was not true for DEE patients $(27 \%$, $8 / 30$ vs. $16 \%, 5 / 32$, adjusted $p=0.5375$; - Fig. 2 ). The proportion of genetic variants of uncertain significance was more than tenfold increased from $5 \%$ in small panels to $52 \%$ in large panels (adjusted $p$-value $=0.0006$ ).

\section{Discussion}

NGS is a proven method to identify monogenetic causes of pediatric epilepsy syndromes. The present study aimed to analyze putative differences in yield of small and large scale customized epilepsy panels in a pediatric population with epilepsy. The borders drawn between small (panels comprising less than $25 \mathrm{~kb}$ of coding DNA) and large (panels comprising more than $25 \mathrm{~kb}$ of coding DNA) panels may seem to be arbitrary. Eventually, this stratification is used by public German Health Insurances as they usually cover only the costs of small panels. Besides this arbitrary segregation, we believed that it is worthwhile to investigate the diagnostic yield of these cohorts separately as data on the influence of the numbers of genes tested in NGS panels on the diagnostic yield are sparse. In addition, previous studies examining the diagnostic yield of various differently sized panels did not cover NGS panels of $25 \mathrm{~kb}$ or less but only larger panels (-Table 2). Thus, it has remained unclear so far if NGS panels below $25 \mathrm{~kb}$ can keep up in efficiency with larger panels.

We analyzed NGS outcomes of 190 patients with either suspected DEE or other less severe types of epilepsy (nonDEE). For all patients in total and for each cohort separately the diagnostic yield of small $(<25 \mathrm{~kb})$ and large panels ( $>25 \mathrm{~kb}$ ) was compared. In our study, for all 190 patients in total, the diagnostic yield of large panels comprising more than $25 \mathrm{~kb}$ (corresponding to a mean of 85.8 genes) was significantly increased compared with smaller panels of less than $25 \mathrm{~kb}$ (corresponding to a mean of 6.4 genes). It is difficult to compare our results directly to previous studies and to compare these studies with each other due to different study designs with different cohorts and panels. However, there seems to be a trend that the range of the diagnostic yield is higher in larger panels. Studies on larger NGS panels of 60 genes and more have shown yields of 20 to $48 \%$ and panels below this number of genes showed pathogenic variants in 18 to $32 \%$ of the patients (-Table 2 ), which is in line with our findings. 


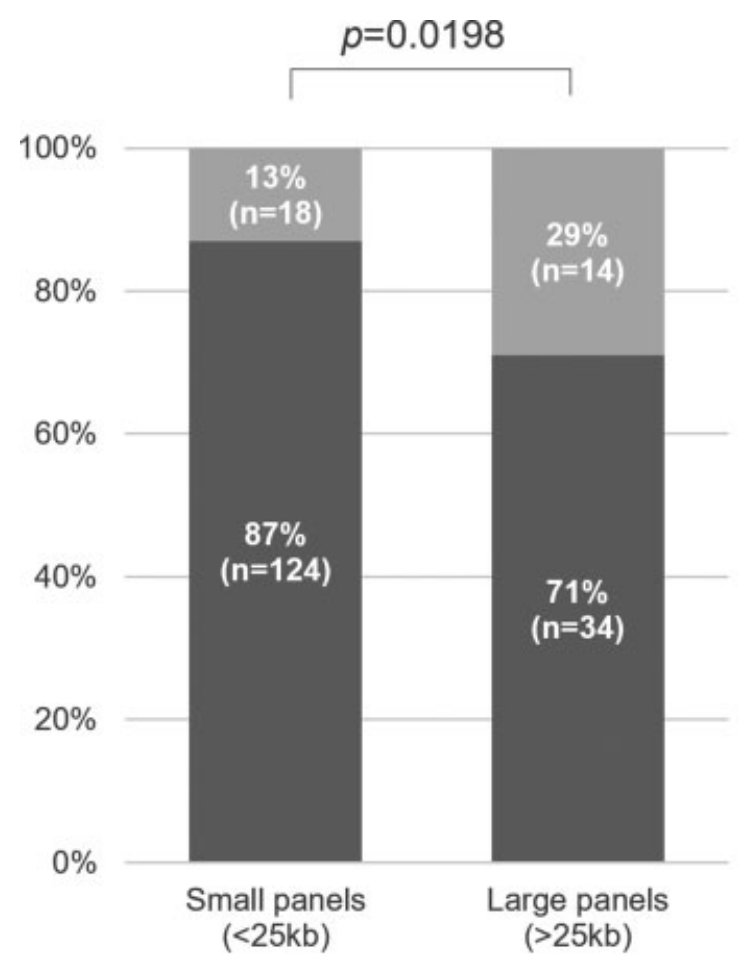

Likely pathogenic and pathogenic variant

No gene affected, likely benign, benign variant or variant of uncertain significance

Fig. 1 Comparing the proportion of likely pathogenic/pathogenic variants in NGS results of small and large panels for all patients in total. NGS, next generation sequencing.

Possible criteria for preselecting the best panel size for a patient is the underlying suspected type of epilepsy. We found that large panels are significantly beneficial compared with small panels for pediatric patients with a non-DEE type of epilepsy ( 35 vs. 13\%). Nevertheless, most data on the yield of customized epilepsy panels consider mainly patients with DEE. A study using a panel comprising 172 genes tested on 278 patients with DEE revealed a yield of pathogenic or likely pathogenic variants in $37.1 \%$ of the cases. ${ }^{4} \mathrm{~A}$ further study tested an NGS panel comprising 106 genes on 87 patients with DEE and found a diagnostic yield of $20 \%$. Panels with less than 60 genes were investigated in a study testing a 46 genes panel on 400 children with DEE. Disease-causing pathogenic variants were identified in $18 \%$ of the patients. ${ }^{10} \mathrm{~A}$ further study on 175 children with DEE undergoing a panel of 17 genes showed a diagnostic yield of $32 \% .{ }^{11}$ In our cohort, small panels revealed less diagnostic yield (pathogenic and likely pathogenic results) compared with large panels (16 vs. 27\%) though these differences did not reveal statistical significance for the DEE group. Thus, small panels of a maximum of $25 \mathrm{~kb}$ of investigated coding DNA seem to be sufficient to yield in comparable amounts of pathogenic or likely pathogenic results to larger panels in patients with DEE which might be an issue when genetic testing is performed in an under resourced health system.

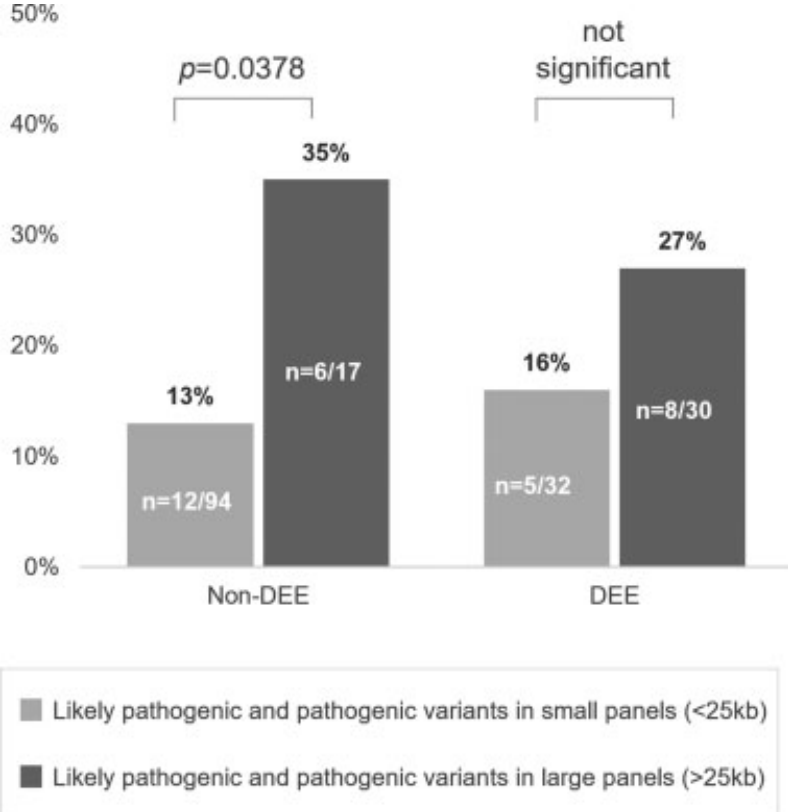

Fig. 2 Proportion of likely pathogenic/pathogenic variants in NGS results of small and large panels comparing patients with developmental and epileptic encephalopathy (DEE) and without DEE (nonDEE). NGS, next generation sequencing.

Coinciding with this result, a previous study that tested various panels ranging from 35 to 265 genes in patients with DEE concluded that panels with 38 genes would have been sufficient to achieve $93 \%$ of the diagnostic outcomes. ${ }^{2}$ A similar result was found in a study for patients with drug-resistant epilepsy. In this study by Parrini et $\mathrm{al}^{12}{ }^{12} 349$ epilepsy patients were simultaneously tested with two NGS panels: a 30-genes panel and a 95-genes panel. Ninety-four percent of all pathogenic variants were found by both panels, only for $6 \%$ of the genetic diagnoses (i.e., for 22 patients) the larger panel detected the pathogenic variant exclusively. Thus, both studies approve our result that in certain cases larger NGS panels are not significantly superior to smaller ones.

Despite the higher benefit of large panels for non-DEE patients in our study, the proportion of patients obtaining a large panel diagnostic was higher in DEE patients compared with non-DEE patients (49 vs. $15 \%$ ). A possible explanation for this result might be the misconception of testing more genes in patients with more severe epilepsy. In contrary, phenotypes in DEE patients tend to be more distinct, which should help to limit the testing of the number of potentially relevant genes. ${ }^{17}$

The portion of VUS was more than tenfold increased in large panels compared with small panels in the present cohort. Rising numbers of genes tested increase the likelihood to find a pathogenic gene variation but they also cause higher costs and disproportionately increase the likelihood to find VUS. ${ }^{18}$ Detection of VUS may make patients insecure and may require further testing of the patient's parents to interpret the variant. However, with ongoing gene discovery and interpretation, it can be assumed that the amount of VUS will decrease. A smaller panel with strictly and phenotype- 
Table 2 Literature review of former studies analyzing the yield of next generation sequencing in pediatric epilepsy patients

\begin{tabular}{|l|l|c|c|c|}
\hline Author (Year) & Epilepsy & Pediatric patients ( $\boldsymbol{n}$ ) & Genes $(\boldsymbol{n})$ & Yield \\
\hline Ko et al (2018) & DEE & 278 & 172 & $37 \%$ \\
\hline Ortega-Moreno (2017) & DEE & 87 & 106 & $20 \%$ \\
\hline Parrini et al (2017) & Drug-resistant epilepsy & 349 & 30 and 95 & 21 and $27 \%$ \\
\hline Zhang et al (2017) & DEE & 175 & 17 & $32 \%$ \\
\hline Segal (2016) & Drug-resistant epilepsy & 49 & $\begin{array}{c}1-81 \\
\text { (several panels) }\end{array}$ & $14 \%$ \\
\hline Trump et al (2016) & DEE & 400 & 46 & $18 \%$ \\
\hline $\begin{array}{l}\text { Mercimek-Mahmutoglu } \\
\text { et al (2015) }\end{array}$ & DEE & 110 & $35-265$ (several panels) & $13 \%$ \\
\hline Della-Mina (2014) & Epilepsy & 19 & 67 & $47 \%$ \\
\hline Wang (2014) & Epilepsy & 28 & 38 and 53 & $21 \%$ \\
\hline Lemke et al (2012) & Epilepsy & 33 & 265 & $48 \%$ \\
\hline
\end{tabular}

Abbreviation: DEE, developmental and epileptic encephalopathy.

related selected genes can increase the chances to detect a disease-causing pathogenic variant by keeping the occurrence of VUS low at the same time. ${ }^{19}$

In the present study, we examined the differences in yield of small versus large scale gene panels in epileptic patients under the special consideration that the Public German Health System does only cover small scale panels at first instance. Large scale panels can be done after special negations with the public insurance, in the majority of private insurances and on research basis. Besides targeted gene sequencing using personalized panels in patients with epilepsy, more advanced techniques as exome, transcriptome, and genome sequencing are available. ${ }^{20,21}$ These techniques may not provide a higher yield of pathogenic or likely pathogenic variants compared with targeted panels but may detect more likely ultrarare variants or previously unrecognized epilepsy-associated genes. ${ }^{22,23}$ In addition, some of these more advanced techniques cover noncoding DNA sequences which also may harbor pathogenic variants leading to epileptic phenotypes.

There are several limitations of this study: (1) Customized epilepsy panels may differ significantly from the genes covered; nevertheless, our results regarding yield were in line with recent other reports. (2) The strict stratification to DEE and non-DEE might appear arbitrary as some patients with non-DEE might reveal significant developmental delay which is not reflected by the EEG as in DEE patients. (3) The segregation of small versus large panels by the amount of tested coding DNA sequence more or less than $25 \mathrm{~kb}$ might be interpreted as also an arbitrary product of the German Health System. Nevertheless, we feel that it was worth to translate these artificial circumstances into a scientific context and analysis. It might encourage under resourced health systems to choose small panels with very carefully selected genes in DEE patients. (4) The sample size of the large and small scale panel cohort varied significantly in this study. Thus, in some cases the statistical significance was just reached. Studies of more comparable sample sizes are needed to further proof these relationships.

\section{Conclusion}

The present study supports the fact that the diagnostic yield of NGS in monogenic epilepsies is increased by the number of genes investigated. In DEE patients, a considerable number of pathogenic and likely pathogenic variants are also found in small customized panels of $25 \mathrm{~kb}$ and less investigated coding DNA. More advanced techniques as exome, transcriptome, and genome investigations may not be superior to customized gene panels with respect to the total yield of proven pathogenic or likely pathogenic results but may more likely detect ultrarare and previously not described genetic variants associated with epilepsy.

Conflict of Interest

None.

\section{References}

1 Chambers C, Jansen LA, Dhamija R. Review of commercially available epilepsy genetic panels. J Genet Couns 2016;25(02):213-217

2 Mercimek-Mahmutoglu S, Patel J, Cordeiro D, et al. Diagnostic yield of genetic testing in epileptic encephalopathy in childhood. Epilepsia 2015;56(05):707-716

3 Richards S, Aziz N, Bale S, et al; ACMG Laboratory Quality Assurance Committee. Standards and guidelines for the interpretation of sequence variants: a joint consensus recommendation of the American College of Medical Genetics and Genomics and the Association for Molecular Pathology. Genet Med 2015;17(05):405-424

4 Ko A, Youn SE, Kim SH, et al. Targeted gene panel and genotypephenotype correlation in children with developmental and epileptic encephalopathy. Epilepsy Res 2018;141:48-55

5 McTague A, Howell KB, Cross JH, Kurian MA, Scheffer IE. The genetic landscape of the epileptic encephalopathies of infancy and childhood. Lancet Neurol 2016;15(03):304-316

6 Berg AT, Berkovic SF, Brodie MJ, et al. Revised terminology and concepts for organization of seizures and epilepsies: report of the ILAE Commission on Classification and Terminology, 2005-2009. Epilepsia 2010;51(04):676-685

7 Stark Z, Schofield D, Alam K, et al. Prospective comparison of the cost-effectiveness of clinical whole-exome sequencing with that of usual care overwhelmingly supports early use and reimbursement. Genet Med 2017;19(08):867-874 
8 Hoelz H, Herdl C, Gerstl L, et al. Impact on clinical decision making of next-generation sequencing in pediatric epilepsy in a tertiary epilepsy referral center. Clin EEG Neurosci 2020;51(01): 61-69

9 Benjamini Y, Hochberg Y. Controlling the false discovery rate: a practical and powerful approach to multiple testing. J R Stat Soc B 1995;57(01):289-300

10 Trump N, McTague A, Brittain $\mathrm{H}$, et al. Improving diagnosis and broadening the phenotypes in early-onset seizure and severe developmental delay disorders through gene panel analysis. J Med Genet 2016;53(05):310-317

11 Zhang Q, Li J, Zhao Y, Bao X, Wei L, Wang J. Gene mutation analysis of 175 Chinese patients with early-onset epileptic encephalopathy. Clin Genet 2017;91(05):717-724

12 Parrini E, Marini C, Mei D, et al; Clinical Study Group. Diagnostic targeted resequencing in 349 patients with drug-resistant pediatric epilepsies identifies causative mutations in 30 different genes. Hum Mutat 2017;38(02):216-225

13 Ortega-Moreno L, Giraldez BG, Soto-Insuga V, et al. Molecular diagnosis of patients with epilepsy and developmental delay using a customized panel of epilepsy genes. PloS one 2017;12 (11): 0188978

14 Segal E, Pedro H, Valdez-Gonzalez K, et al. Diagnostic yeld of epilepsy panels in children with medication-refractory epilepsy. Pediatr Neurol 2016;64:66-71
15 Della Mina E, Ciccone R, Brustia F, et al. Improving molecular diagnosis in epilepsy by a dedicated high-throughput sequencing platform. Eur J of Hum Genet 2015;23(3):354-362

16 Wang J, Gotway G, Pascual JM, Park JY. Diagnostic yield of clinical next-generation sequencing panels for epilepsy. JAMA Neurol 2014;71(5):650-651

17 Lemke JR, Riesch E, Scheurenbrand T, et al. Targeted next generation sequencing as a diagnostic tool in epileptic disorders. Epilepsia 2012;53(08):1387-1398

18 Ream MA, Patel AD. Obtaining genetic testing in pediatric epilepsy. Epilepsia 2015;56(10):1505-1514

19 Dunn P, Albury CL, Maksemous N, et al. Next generation sequencing methods for diagnosis of epilepsy syndromes. Front Genet 2018;9:20

20 Snoeijen-Schouwenaars FM, van Ool JS, Verhoeven JS, et al. Diagnostic exome sequencing in 100 consecutive patients with both epilepsy and intellectual disability. Epilepsia 2019;60(01):155-164

21 Heyne HO, Singh T, Stamberger H, et al; EuroEPINOMICS RES Consortium. De novo variants in neurodevelopmental disorders with epilepsy. Nat Genet 2018;50(07):1048-1053

22 Heyne HO, Artomov M, Battke F, et al. Targeted gene sequencing in 6994 individuals with neurodevelopmental disorder with epilepsy. Genet Med 2019;21(11):2496-2503

23 Ultra-Rare Genetic Variation in the Epilepsies: A Whole-Exome Sequencing Study of 17,606 Individuals. Am J Hum Genet 2019;105(2):267-282 